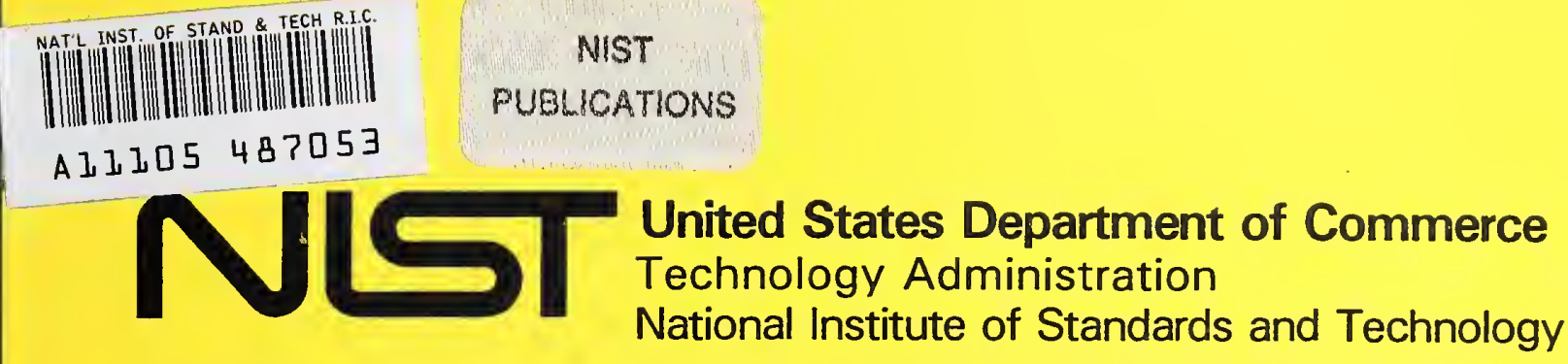

NISTIR 5074

Rapid Evaluation of Nonreflecting Boundary Kernels for Time-Domain Wave Propagation

Bradley Alpert

Leslie Greengard

Thomas Hagstrom

QC

100

.056

N0.5074

1998 



\title{
Rapid Evaluation of Nonreflecting Boundary Kernels for Time-Domain Wave Propagation
}

\author{
Bradley Alpert \\ Leslie Greengard \\ Thomas Hagstrom
}

Mathematical and Computational Sciences Division

Information Technology Laboratory

National Institute of Standards and Technology

Boulder, Colorado 80303-3328

April 1998

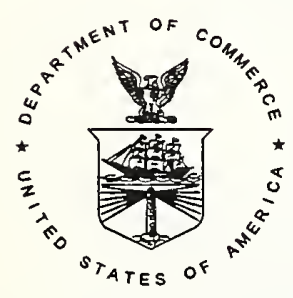

U.S. DEPARTMENT OF COMMERCE, William M. Daley, Secretary TECHNOLOGY ADMINISTRATION, Gary R. Bachula, Acting Under Secretary for Technology NATIONAL INSTITUTE OF STANDARDS AND TECHNOLOGY, Raymond G. Kammer, Director 



\title{
RAPID EVALUATION OF NONREFLECTING BOUNDARY KERNELS FOR TIME-DOMAIN WAVE PROPAGATION
}

\author{
BRADLEY ALPERT \\ Mathematical and Computational Sciences Division \\ National Institute of Standards and Technology, Boulder, Colorado 80303 \\ LESLIE GREENGARD \\ Courant Institute of Mathematical Sciences \\ New York University, 251 Mercer Street, New York, NY 10012-1110 \\ THOMAS HAGSTROM \\ Department of Mathematics and Statistics, \\ University of New Mexico, Albuquerque, NM 87131
}

\begin{abstract}
We present a systematic approach to the computation of exact nonreflecting boundary conditions for the wave equation. In both two and three dimensions, the critical step in our analysis involves convolution with the inverse Laplace transform of the logarithmic derivative of a Hankel function. The main technical result in this paper is that the logarithmic derivative of the Hankel function $H_{v}^{(1)}(z)$ of real order $v$ can be approximated in the upper half $z$-plane with relative error $\varepsilon$ by a rational function of degree $d \sim O\left(\log |v| \log \frac{1}{\varepsilon}+\log ^{2}|v|+|\nu|^{-1} \log ^{2} \frac{1}{\varepsilon}\right)$ as $|\nu| \rightarrow \infty, \varepsilon \rightarrow 0$, with slightly more complicated bounds for $v=0$. If $N$ is the number of points used in the discretization of a cylindrical (circular) boundary in two dimensions, then, assuming that $\varepsilon<1 / N$, $O\left(N \log N \log \frac{1}{\varepsilon}\right)$ work is required at each time step. This is comparable to the work required for the Fourier transform on the boundary. In three dimensions, the cost is proportional to $N^{2} \log ^{2} N+N^{2} \log N \log \frac{1}{\varepsilon}$, for a spherical boundary with $N^{2}$ points, the first term coming from the calculation of a spherical harmonic transform at each time step. In short, nonreflecting boundary conditions can be imposed to any desired accuracy, at a cost dominated by the interior grid work, which scales like $N^{2}$ in two dimensions and $N^{3}$ in three dimensions.
\end{abstract}

KEY WORDS. Bessel function, approximation, high-order convergence, wave equation, Maxwell's equations, nonreflecting boundary condition, radiation boundary condition, absorbing boundary condition

E-mail addresses: alpert@boulder.nist.gov,greengar@cims.nyu.edu,hagstrom@math.unm.edu.

Alpert supported in part by DARPA under appropriation 9770400. Greengard and Hagstrom supported in part by the U.S. Department of Energy under contract DE-FGO288ER25053 and DARPA/AFOSR under contract F94620-95-C-0075. Hagstrom also supported in part by National Science Foundation under grant DMS-9600146. 


\section{INTRODUCTION}

A longstanding practical issue in numerical wave propagation and scattering problems concerns the reduction of an unbounded domain to a bounded domain by the imposition of nonreflecting boundary conditions at an artificial boundary. We restrict our attention to "time-domain" calculations, for which it is well-known that the exact nonreflecting conditions are global in both space and time. While the problem has been widely studied (see Givoli [1] for an overview), the boundary conditions used in practice typically introduce serious numerical artifacts. The two most common approaches are based on the construction of local differential boundary conditions $[2,3]$ or absorbing regions $[4,5]$, but neither provides a clear sequence of approximations which converge to the exact, nonlocal conditions. Recently, Sofronov [6] and, independently, Grote and Keller [7] have developed and implemented an integrodifferential approach for three-dimensional calculations using a spherical boundary and have demonstrated that high accuracy can be achieved at reasonable cost. In their schemes, the work is of the same order as the explicit finite difference or finite element calculation in the interior of the domain. For $N^{2}$ points on the spherical boundary, $O\left(N^{3}\right)$ work is required. Hagstrom and Hariharan [8] have shown that these conditions can be effectively implemented using only local operators, but at the cost of introducing a large number of auxiliary functions at the boundary. A somewhat more general, but closely related, integral formulation is introduced in $[9,10]$. The fundamental analytical tool in the latter papers is what we refer to as the "nonreflecting boundary kernel" which is the inverse Laplace transform of the logarithmic derivative of a Hankel function.

In this paper, we prove that the logarithmic derivative of a Hankel function can be approximated as a ratio of polynomials of modest degree, so that its inverse Laplace transform can be expressed as a sum of exponentials. Our analytical approach combines an extension of the Mittag-Leffler theorem with the approximation techniques of the fast multipole method. In particular, Theorem 4.1 presents an exact representation of the logarithmic derivative as a sum of poles plus a continuous density on the branch cut. Theorem 4.6 , which is preceded by several technical lemmas, presents a reduced, approximate representation.

Using this approach, the cost of computing the nonreflecting boundary condition is comparable to that of a fast Fourier or spherical harmonic transform. For two-dimensional problems, $O\left(N \log N \log \frac{1}{\varepsilon}\right)$ work is required at each time step, where $N$ is the number of points used in the discretization of a cylindrical (circular) boundary. In three dimensions, the cost is proportional to $N^{2} \log ^{2} N+N^{2} \log N \log \frac{1}{\varepsilon}$, for a spherical boundary with $N^{2}$ points. The first term comes from the calculation of the spherical harmonic transform using the fast algorithm of $[11,12]$.

Other authors, including Nédélec [13] and Cruz and Sesma [14], have studied the logarithmic derivative of the Hankel function, based on a variety of techniques. In this paper we present a sum-of-poles representation for the logarithmic derivative of a Hankel function of real order $v$ bounded away from zero with accuracy $\varepsilon$ for argument, $z$, satisfying $\operatorname{Im}(z) \geq 0$. The number of poles is bounded by $O\left(\log |\nu| \cdot \log \frac{1}{\varepsilon}+\log ^{2}|v|+|v|^{-1} \log ^{2} \frac{1}{\varepsilon}\right)$. A similar representation for $v=0$ is also derived which is valid for $\operatorname{Im}(z) \geq \eta>0$ requiring $O\left(\log \frac{1}{\eta} \cdot \log \frac{1}{\varepsilon}+\log \frac{1}{\varepsilon} \cdot \log \log \frac{1}{\varepsilon}+\log \frac{1}{\eta} \cdot \log \log \frac{1}{\eta}\right)$ poles. 
In Section 2, we introduce nonreflecting boundary kernels. In Section 3 we collect background material in a form convenient for the subsequent development. Section 4 contains the analytical and approximate treatment of the logarithmic derivative, while a procedure for computing these representations is presented in Section 5. The results of our numerical computations are contained in Section 6, and we present our conclusions in Section 7.

\section{NONREFLECTING BOUNDARY KERNELS}

Let us first consider the wave equation

$$
u_{t t}=c^{2} \nabla^{2} u
$$

in a two-dimensional annular domain $\rho_{0}<\rho<\rho_{1}$. The general solution can be expressed as

$$
u(\rho, \phi, t)=\sum_{n=-\infty}^{\infty} e^{i n \phi} \mathcal{L}^{-1}\left[a_{n}(s) K_{n}(\rho s / c)+b_{n}(s) I_{n}(\rho s / c)\right](t),
$$

where $K_{n}$ and $I_{n}$ are modified Bessel functions (see, for example, [15] 9.6),

$$
K_{n}(z)=\frac{\pi}{2} i^{n+1} H_{n}^{(1)}\left(z e^{\pi i / 2}\right), \quad I_{n}(z)=i^{-n} J_{n}\left(z e^{\pi i / 2}\right), \quad-\pi<\arg z \leq \frac{\pi}{2},
$$

the coefficients $a_{n}$ and $b_{n}$ are arbitrary functions analytic in the right half plane, $\mathcal{L}$ denotes the Laplace transform

$$
\mathcal{L}[f](s)=\int_{0}^{\infty} e^{-s t} f(t) d t
$$

and $\mathcal{L}^{-1}$ denotes the inverse Laplace transform

$$
\mathcal{L}^{-1}[g](t)=\frac{1}{2 \pi i} \int_{-i \infty}^{i \infty} e^{s t} g(s) d s .
$$

Likewise, for the wave equation in a three-dimensional domain $r_{0}<r<r_{1}$, the general solution can be expressed as

$$
\begin{aligned}
u(r, \phi, \theta, t)=\sum_{n=-\infty}^{\infty} \sum_{m=-n}^{n} Y_{n m}(\phi, \theta) \mathcal{L}^{-1} & {\left[a_{n m}(s) \frac{K_{n+\frac{1}{2}}(r s / c)}{\sqrt{r s / c}}\right.} \\
& \left.+b_{n m}(s) \frac{I_{n+\frac{1}{2}}(r s / c)}{\sqrt{r s / c}}\right](t) .
\end{aligned}
$$

If we imagine that $\rho=\rho_{1}$ (or $r=r_{1}$ ) is to be used as a nonreflecting boundary, then we can assume there are no sources in the exterior region and the coefficients $b_{n}(s)$ (or $b_{n m}(s)$ ) are zero. Let us now denote by $u_{n}(\rho, t)$ the function satisfying

$$
\mathcal{L}\left[u_{n}\right](\rho, s)=a_{n}(s) K_{n}(\rho s / c) .
$$


Then

so that

$$
\begin{aligned}
\mathcal{L}\left[\frac{\partial}{\partial \rho} u_{n}\right](\rho, s) & =a_{n}(s) \cdot \frac{s}{c} \cdot K_{n}^{\prime}(\rho s / c) \\
& =\mathcal{L}\left[u_{n}\right](\rho, s) \cdot\left(\frac{s}{c} \frac{K_{n}^{\prime}(\rho s / c)}{K_{n}(\rho s / c)}\right)
\end{aligned}
$$

where $*$ denotes Laplace convolution

$$
\frac{\partial}{\partial \rho} u_{n}(\rho, t)=u_{n}(\rho, t) * \mathcal{L}^{-1}\left[\frac{s}{c} \frac{K_{n}^{\prime}(\rho s / c)}{K_{n}(\rho s / c)}\right](t),
$$

$$
(f * g)(t)=\int_{0}^{t} f(\tau) g(t-\tau) d \tau
$$

The convolution kernel in (9) is a generalized function. Its singular part is easily removed, however, by subtracting the first two terms of the asymptotic expansion

$$
\frac{s}{c} \frac{K_{n}^{\prime}(\rho s / c)}{K_{n}(\rho s / c)} \sim-\frac{s}{c}-\frac{1}{2 \rho}+O\left(s^{-1}\right), \quad s \rightarrow \infty .
$$

From the assumption $u_{n}(\rho, t)=0$ for $t \leq 0$ and standard properties of the Laplace transform we obtain the boundary condition

$$
\frac{\partial}{\partial \rho} u_{n}(\rho, t)+\frac{1}{c} \frac{\partial}{\partial t} u_{n}(\rho, t)+\frac{1}{2 \rho} u_{n}(\rho, t)=\int_{0}^{t} \sigma_{n}(t-\tau) u_{n}(\rho, \tau) d \tau,
$$

where

$$
\sigma_{n}(t)=\mathcal{L}^{-1}\left[\frac{s}{c}+\frac{1}{2 \rho}+\frac{s}{c} \frac{K_{n}^{\prime}(\rho s / c)}{K_{n}(\rho s / c)}\right](t)
$$

which we impose at $\rho=\rho_{1}$.

Remark. The solution to the wave equation in physical space is recovered on the nonreflecting boundary from $u_{n}$ by Fourier transformation:

$$
u\left(\rho_{1}, \phi, t\right)=\sum_{n=-N / 2}^{N / 2-1} u_{n}\left(\rho_{1}, t\right) e^{i n \phi}
$$

assuming $N$ points are used in the discretization.

The analogous boundary condition in three dimensions is expressed in terms of the functions $u_{n m}(r, t)$ satisfying

$$
\mathcal{L}\left[u_{n m}\right](r, s)=a_{n m}(s) \frac{K_{n+\frac{1}{2}}(r s / c)}{\sqrt{r s / c}} .
$$

After some algebraic manipulation, assuming $u_{n m}(r, t)=0$ for $t \leq 0$, we have

$$
\frac{\partial}{\partial r} u_{n m}(r, t)+\frac{1}{c} \frac{\partial}{\partial t} u_{n m}(r, t)+\frac{1}{r} u_{n m}(r, t)=\int_{0}^{t} \omega_{n}(t-\tau) u_{n m}(r, \tau) d \tau,
$$


where

$$
\omega_{n}(t)=\mathcal{L}^{-1}\left[\frac{s}{c}+\frac{1}{2 r}+\frac{s}{c} \frac{K_{n+\frac{1}{2}}^{\prime}(r s / c)}{K_{n+\frac{1}{2}}(r s / c)}\right](t)
$$

which we impose at $r=r_{1}$.

Note that the boundary conditions (12) and (16) are exact but nonlocal, since they rely on a Fourier (or spherical harmonic) transformation in space, and are history dependent. The form of the history is simple, however, and expressed, for each separate mode, in terms of a convolution kernel which is the inverse Laplace transform of a function defined in terms of the logarithmic derivative of a modified Bessel function

$$
\frac{d}{d z} \log K_{v}(z)=\frac{K_{v}^{\prime}(z)}{K_{v}(z)}
$$

Remark. In three dimensions, the required logarithmic derivative of $K_{n+\frac{1}{2}}(z)$ is a ratio of polynomials, so that one can recast the boundary condition in terms of a differential operator of order $n$. The resulting expression would be equivalent to those derived by Sofronov [6] and Grote and Keller [7].

The remainder of this paper is devoted to the approximation of the logarithmic derivatives $(18)$ as a ratio of polynomials of degree $O(\log \nu)$, from which the convolution kernels $\sigma_{n}$ and $\omega_{n}$ can be expressed as a sum of decaying exponentials. This representation allows for the recursive evaluation of the integral operators in (12) and (16), using only $O(\log n)$ work per time step (see [16]). We note that, by Parseval's equality, the $L_{2}$ error resulting from convolution with an approximate kernel is sharply bounded by the $L_{\infty}$ error in the approximation to the kernel's transform. Precisely, approximating the kernel $B(t)$ by the kernel $A(t)$ we find

$$
\begin{aligned}
\|A * u-B * u\|_{2}=\|\hat{A} \hat{u}-\hat{B} \hat{u}\|_{2} & \leq \sup _{s \in i \mathbb{R}} \frac{|\hat{A}-\hat{B}|}{|\hat{B}|}\|\hat{B} \hat{u}\|_{2} \\
& =\sup _{s \in i \mathbb{R}} \frac{|\hat{A}-\hat{B}|}{|\hat{B}|}\|B * u\|_{2},
\end{aligned}
$$

where we assume that $\hat{A}, \hat{B}$, and $\hat{u}$ are all regular for $\operatorname{Re}(s)>0$. For finite times we may let $s$ have a positive real part, $\eta$ :

$$
\|A * u-B * u\|_{L_{2}(0, T)} \leq e^{\eta T} \sup _{s \in \eta+i \mathbb{R}} \frac{|\hat{A}-\hat{B}|}{|\hat{B}|}\|B * u\|_{L_{2}(0, T)} .
$$

We therefore concentrate our theoretical developments on $L_{\infty}$ approximations. For ease of computation, however, we compute our rational representations by least squares methods. These do generally lead to small relative errors in the maximum norm, as will be shown. 
Since Hankel functions are more commonly used in the special function literature, we will write the logarithmic derivatives as

$$
\frac{d}{d z} \log K_{v}(z)=\frac{d}{d z} \log H_{v}^{(1)}\left(z e^{\pi i / 2}\right)=i \frac{H_{v}^{(1)^{\prime}}\left(z e^{\pi i / 2}\right)}{H_{v}^{(1)}\left(z e^{\pi i / 2}\right)} .
$$

We are, then, interested in approximating logarithmic derivative of the Hankel function on and above the real axis.

\section{Mathematical Preliminaries}

In this section we collect several well-known facts concerning the Bessel equation, the logarithmic derivative of the Hankel function, and pole expansions, in a form that will be useful in the subsequent analytical development.

3.1. Bessel's Equation. Bessel's differential equation

$$
\frac{d^{2} u}{d z^{2}}+\frac{1}{z} \frac{d u}{d z}+\left(1-\frac{v^{2}}{z^{2}}\right) u=0,
$$

for $\nu \in \mathbb{R}$, has linearly independent solutions $H_{v}^{(1)}$ and $H_{v}^{(2)}$, known as Hankel's functions. These can be expressed by the formulae

$$
H_{v}^{(1)}(z)=\frac{J_{-v}(z)-e^{-v \pi i} J_{v}(z)}{i \sin (v \pi)}, \quad H_{v}^{(2)}(z)=-\frac{J_{-v}(z)-e^{v \pi i} J_{v}(z)}{i \sin (\nu \pi)},
$$

where the Bessel function of the first kind is defined by

$$
J_{v}(z)=\left(\frac{z}{2}\right)^{v} \sum_{k=0}^{\infty} \frac{\left(-z^{2} / 4\right)^{k}}{k ! \Gamma(\nu+k+1)} .
$$

The expressions in (23) are replaced by their limiting values for integer values of $\nu$. (See, for example, $[15,9.1]$ ) For general $v$, the functions $H_{v}^{(1)}$ and $H_{v}^{(2)}$ have a branch point at $z=0$ and it is customary to place the corresponding branch cut on the negative real axis and impose the restriction $-\pi<\arg z \leq \pi$. We shall find it more convenient, however, to place the branch cut on the negative imaginary axis, with the restriction

$$
-\frac{\pi}{2} \leq \arg z<\frac{3 \pi}{2} \text {. }
$$

Hankel's functions have especially simple asymptotic properties. In particular (see, for example, $[17,7.4 .1])$,

$$
\begin{aligned}
& H_{v}^{(1)}(z) \sim\left(\frac{2}{\pi z}\right)^{1 / 2} e^{i(z-v \pi / 2-\pi / 4)} \sum_{k=0}^{\infty} i^{k} \frac{A_{k}(\nu)}{z^{k}}, \\
& H_{v}^{(1)^{\prime}}(z) \sim\left(\frac{2}{\pi z}\right)^{1 / 2} e^{i(z-v \pi / 2-\pi / 4)} \sum_{k=0}^{\infty} i^{k} \frac{A_{k}(\nu)}{z^{k}}\left(-\frac{1}{2 z}+i-\frac{k}{z}\right),
\end{aligned}
$$


as $z \rightarrow \infty$, with $-\pi+\delta \leq \arg z \leq 2 \pi-\delta$, where

$$
A_{k}(v)=\frac{\left(4 v^{2}-1^{2}\right)\left(4 v^{2}-3^{2}\right) \cdots\left(4 v^{2}-(2 k-1)^{2}\right)}{k ! 8^{k}},
$$

and the branch of the square root is determined by

$$
z^{1 / 2}=e^{(\log |z|+i \arg z) / 2} .
$$

Finally we note the symmetry

$$
H_{v}^{(1)}(z)=e^{-v \pi i} H_{-v}^{(1)}(z) .
$$

We also make use of the modified Bessel functions, $K_{v}(z)$ and $I_{v}(z)$. These are linearly independent solutions of the equation obtained from (22) by the transformation $z \rightarrow i z$. Their Wronskian satisfies

$$
K_{v}(z) I_{v}^{\prime}(z)-K_{v}^{\prime}(z) I_{v}(z)=z^{-1}
$$

Moreover we have for positive $r$ [18]

$$
H_{\nu}^{(1)}\left(r e^{-i \pi / 2}\right)=\frac{2}{\pi i} e^{-v \pi i / 2}\left(e^{\nu \pi i} K_{\nu}(r)+\pi i I_{\nu}(r)\right) .
$$

Asymptotic expansions of $K_{\nu}(r)$ and $I_{\nu}(r)$ for $r$ small and large are also known [15, 9.6, 9.7]. For real $r$ and $\nu \geq 0$ we have

$$
\begin{aligned}
& K_{v}(r) \sim \begin{cases}\gamma-\log \frac{r}{2}, & v=0, \\
\frac{\Gamma(\nu)}{2}\left(\frac{r}{2}\right)^{-v}, & v>0,\end{cases} \\
& I_{\nu}(r) \sim \frac{1}{\Gamma(\nu+1)}\left(\frac{r}{2}\right)^{\nu}, \quad r \rightarrow 0, \\
& K_{v}(r) \sim \sqrt{\frac{\pi}{2 r}} e^{-r}, \quad r \rightarrow \infty, \\
& I_{\nu}(r) \sim \sqrt{\frac{1}{2 \pi r}} e^{r}, \quad r \rightarrow \infty .
\end{aligned}
$$

Here $\gamma=0.5772 \ldots$ is the Euler constant.

Finally, we note the uniform expansions of Bessel functions for $v \rightarrow \infty$ given in [15]. For Hankel function and derivative we have

$$
\begin{aligned}
H_{\nu}^{(1)}(\nu z) & \sim 2 e^{-\pi i / 3}\left(\frac{4 \zeta}{1-z^{2}}\right)^{1 / 4} \frac{\operatorname{Ai}\left(e^{2 \pi i / 3} v^{2 / 3} \zeta\right)}{v^{1 / 3}} \\
H_{\nu}^{(1)^{\prime}}(\nu z) & \sim \frac{4 e^{-2 \pi i / 3}}{z}\left(\frac{4 \zeta}{1-z^{2}}\right)^{-1 / 4} \frac{\mathrm{Ai}^{\prime}\left(e^{2 \pi i / 3} v^{2 / 3} \zeta\right)}{v^{2 / 3}}
\end{aligned}
$$

as $\nu \rightarrow \infty$, where we restrict $z$ to $|\arg (z)| \leq \pi / 2$ and define

$$
\frac{2}{3} \zeta^{3 / 2}=\log \frac{1+\sqrt{1-z^{2}}}{z}-\sqrt{1-z^{2}}
$$


Here, $\operatorname{Ai}(t)$ denotes the Airy function $[15,10.4]$. Note that $\zeta=0$ when $z=1$. Large $v$ approximations of the modified Bessel functions for real arguments, $r$, are given by

$$
K_{v}(v r) \sim \sqrt{\frac{\pi}{2 v}} \frac{e^{-v \phi(r)}}{\left(1+r^{2}\right)^{1 / 4}}, \quad I_{v}(v r) \sim \frac{1}{\sqrt{2 \pi v}} \frac{e^{v \phi(r)}}{\left(1+r^{2}\right)^{1 / 4}}, \quad v \rightarrow \infty,
$$

where

$$
\phi(r)=\log \frac{r}{1+\sqrt{1+r^{2}}}+\sqrt{1+r^{2}} .
$$

3.2. Hankel Function Logarithmic Derivative. We denote the logarithmic derivative of $H_{v}^{(1)}$ by $G_{v}$,

$$
G_{\nu}(z)=\frac{d}{d z} \log H_{\nu}^{(1)}(z)=\frac{H_{\nu}^{(1)^{\prime}}(z)}{H_{\nu}^{(1)}(z)} .
$$

The following lemma states a few fundamental facts about $G_{\nu}$ that we will use below.

Lemma 3.1. The function $G_{\nu}(z)$, for $v \in \mathbb{R}$, satisfies the formulae

$$
\begin{gathered}
G_{-\nu}(z)=G_{\nu}(z), \\
G_{\nu}\left(\bar{z} e^{\pi i}\right)=\overline{G_{\nu}(z)} e^{\pi i}, \quad-\frac{\pi}{2}<\arg z \leq \frac{\pi}{2},
\end{gathered}
$$

where $\bar{z}=|z| e^{-i \arg z}$ is the complex conjugate of $z$. Asymptotic approximations to $G_{\nu}$ are

$$
G_{v}(z) \sim \begin{cases}\left(\log \left(z e^{-\pi i / 2} / 2\right)+\gamma\right)^{-1} z^{-1}+O(z), & v=0, \\ -|\nu| z^{-1}+O\left(z^{2|\nu|-1}\right), & 0<|\nu|<1, \quad z \rightarrow 0, \\ -|\nu| z^{-1}+O(z \log z), & |v|=1, \\ -|v| z^{-1}+O(z), & |v|>1,\end{cases}
$$

where $\gamma$ is the Euler constant,

$$
G_{v}(z) \sim \sum_{k=0}^{\infty} i^{k} \frac{A_{k}(v)}{z^{k}}\left(-\frac{1}{2 z}+i-\frac{k}{z}\right) / \sum_{k=0}^{\infty} i^{k} \frac{A_{k}(v)}{z^{k}}, \quad z \rightarrow \infty,
$$

where $A_{k}(v)$ is defined in (28), and

$$
G_{v}(v z) \sim \frac{2 e^{-\pi i / 3}}{v^{1 / 3} z}\left(\frac{4 \zeta}{1-z^{2}}\right)^{-1 / 2} \frac{\mathrm{Ai}^{\prime}\left(e^{2 \pi i / 3} v^{2 / 3} \zeta\right)}{\operatorname{Ai}\left(e^{2 \pi i / 3} v^{2 / 3} \zeta\right)}, \quad v \rightarrow \infty,
$$

where $\zeta$ is defined in (39). Furthermore, the function $u_{v}$ defined by

$$
u_{v}(z)=z G_{v}(z)
$$

satisfies the recurrence

$$
u_{v}(z)=\frac{z^{2}}{v-1-u_{v-1}(z)}-v
$$




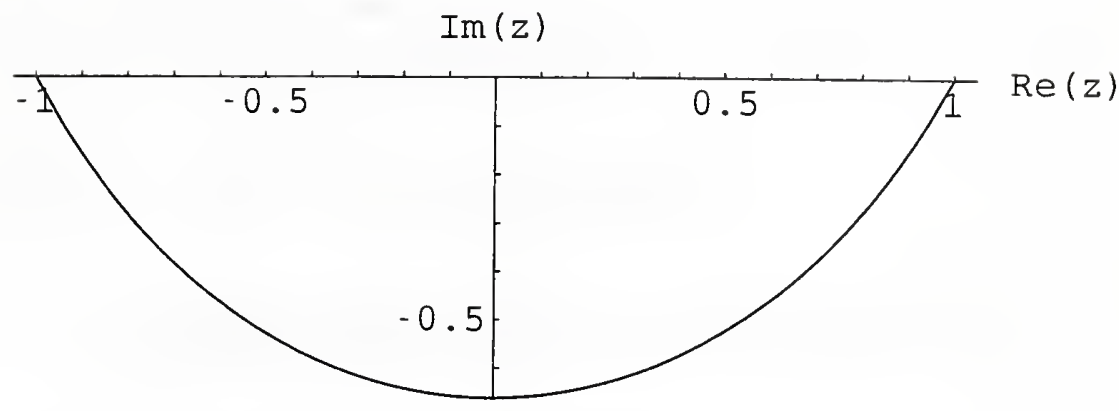

FIGURE 1. Curve $z(\zeta)$ defined by (39) near which the scaled zeros of $H_{\nu}^{(1)}$ lie (see Lemma 3.2). The branch cut of $H_{v}^{(1)}$ is chosen (25) on the negative imaginary axis.

Proof. Equations (43) and (44) and asymptotic expansion (45) follow immediately from the definitions (23) through (25) of $J_{v}$ and $H_{v}^{(1)}$. The asymptotic expansion (46) follows from (26) and (27), while (47) is a consequence of (37) and (38). The recurrence (49) is derived from standard Bessel recurrences [15, 9.1.27].

The zeros of $H_{\nu}^{(1)}(z)$ are well characterized [15, 18]; they lie in the lower half $z$-plane near the curve shown in Figure 1 obtained by transformation [19] of Bessel's equation. In terms of the asymptotic approximation (37), this curve corresponds to negative, real arguments of the Airy function.

Lemma 3.2. The zeros $h_{v, 1}, h_{v, 2}, \ldots$ of $H_{v}^{(1)}(z)$ in the sector $-\pi / 2 \leq \arg z \leq 0$ are given by the asymptotic expansion

$$
\begin{array}{ll}
h_{v, n} \sim v z\left(\zeta_{n}\right)+O\left(v^{-1}\right), & \\
v & n=1, \ldots,\lfloor|v| / 2+1 / 4\rfloor,
\end{array}
$$

uniformly in $n$, where $\zeta_{n}$ is defined by the equation

$$
\zeta_{n}=e^{-2 \pi i / 3} v^{-2 / 3} a_{n}
$$

$z(\zeta)$ is obtained from inverting (39), and $a_{n}$ is the nth negative zero of Airy function Ai. The zeros in the sector $\pi \leq \arg z \leq 3 \pi / 2$ are given by $-\overline{h_{v, 1}},-\overline{h_{v, 2}}, \ldots$ In particular,

$$
h_{v, 1} \sim \nu+e^{-2 \pi i / 3}(\nu / 2)^{1 / 3}\left(-a_{1}\right),
$$

where $-a_{1}=2.338 \ldots$

3.3. Pole Expansions. A set of poles in a finite region defines a function that is smooth away from the region, with the smoothness increasing as the distance increases. This fact leads to the following approximation related to the fast multipole method [20, 21]. 
Lemma 3.3. Suppose that $q_{1}, \ldots, q_{n}$ are complex numbers and $z_{1}, \ldots, z_{n}$ are complex numbers with $\left|z_{j}\right| \leq 1$ for $j=1, \ldots, n$. The function

$$
f(z)=\sum_{j=1}^{n} \frac{q_{j}}{z-z_{j}}
$$

can be approximated for $\operatorname{Re}(z)=a>1$ by the $m$ pole expansion

$$
g_{m}(z)=\sum_{j=0}^{m-1} \frac{\gamma_{j}}{z-\omega^{j}}
$$

where $\omega=e^{2 \pi i / m}$ is a root of unity and $\gamma_{j}$ is defined by

$$
\gamma_{j}=\frac{1}{m} \sum_{l=0}^{m-1} \omega^{-j l} \sum_{k=1}^{n} q_{k} z_{k}^{l}, \quad j=0, \ldots, m-1 .
$$

The error of the approximation is bounded by

$$
\left|f(z)-g_{m}(z)\right| \leq \frac{2\left(a^{2}+1\right)}{\left(a^{m}-1\right)(a-1)^{2}}|F(z)|,
$$

where

$$
F(z)=\sum_{j=1}^{n} \frac{\left|q_{j}\right|}{z-z_{j}} .
$$

Proof. We use the geometric series summation

$$
\frac{1}{z-v}=\sum_{k=0}^{m-1} \frac{v^{k}}{z^{k+1}}+\frac{v^{m}}{z^{m}} \frac{1}{z-v}
$$

to obtain

$$
\begin{aligned}
f(z)-g_{m}(z)= & \sum_{k=0}^{m-1} \frac{1}{z^{k+1}}\left(\sum_{j=1}^{n} q_{j} z_{j}^{k}-\sum_{j=0}^{m-1} \gamma_{j} \omega^{j k}\right) \\
& +\frac{1}{z^{m}}\left(\sum_{j=1}^{n} \frac{q_{j} z_{j}^{m}}{z-z_{j}}-\sum_{j=0}^{m-1} \frac{\gamma_{j} \omega^{j m}}{z-\omega^{j}}\right) .
\end{aligned}
$$


All $m$ terms of the first summation vanish, due to the combination of (55) and the equality $\sum_{j=0}^{m-1} \omega^{j k}=m \delta_{k 0}$. For the error term we obtain

$$
\begin{aligned}
& \left|\sum_{j=1}^{n} \frac{q_{j} z_{j}^{m}}{z-z_{j}}\right| \leq \sum_{j=1}^{n}\left|\frac{q_{j} z_{j}^{m}}{z-z_{j}}\right| \leq \frac{1}{|z|} \sum_{j=1}^{n} \frac{\left|q_{j}\right|}{\left|1-z_{j} / z\right|} \\
& \quad \leq \frac{1}{|z|} \frac{a^{2}+1}{(a-1)^{2}} \sum_{j=1}^{n} \frac{\left(1-a^{-1}\right)\left|q_{j}\right|}{1+a^{-2}} \leq \frac{a^{2}+1}{(a-1)^{2}} \frac{1}{|z|} \operatorname{Re}\left(\sum_{j=1}^{n} \frac{\left|q_{j}\right|}{1-z_{j} / z}\right) \\
& \quad \leq \frac{a^{2}+1}{(a-1)^{2}}\left|\sum_{j=1}^{n} \frac{\left|q_{j}\right|}{z-z_{j}}\right|=\frac{a^{2}+1}{(a-1)^{2}}|F(z)|,
\end{aligned}
$$

and

$$
\left|\sum_{j=0}^{m-1} \frac{\gamma_{j} \omega^{j m}}{z-\omega^{j}}\right|=\left|\sum_{j=0}^{m-1} \frac{\gamma_{j}}{z-\omega^{j}}\right|=\left|g_{m}(z)\right| .
$$

Moreover, repeating the computations of $(60)$, we find

$$
|f(z)| \leq \frac{a^{2}+1}{(a-1)^{2}}|F(z)|
$$

Now the combination of (59) through (62) and the triangle inequality gives (56).

Inequality (56) remains valid if we assume instead that $\left|z_{j}\right| \leq b$ and $\operatorname{Re}(z)=a b>b$, for arbitrary $b \in \mathbb{R}, b>0$; this fact leads to the next two results whose proofs mimic that of Lemma 3.3 and are omitted.

Lemma 3.4. Suppose $n, p$ are positive integers, $q_{1}, \ldots, q_{n}$ are complex numbers, and $z_{1}, \ldots, z_{n}$ are complex numbers contained in disks $D_{1}, \ldots, D_{p}$ of radii $r_{1}, \ldots, r_{p}$, centered at $c_{1}, \ldots, c_{p}$, respectively. The function

$$
f(z)=\sum_{j=1}^{n} \frac{q_{j}}{z-z_{j}}
$$

can be approximated for $z$ satisfying $\operatorname{Re}\left(z-c_{i}\right) \geq a r_{i}>r_{i}$ for $i=1, \ldots, p$ by the $m \cdot p$ pole expansion

$$
g_{m}(z)=\sum_{i=1}^{p} \sum_{j=0}^{m-1} \frac{\gamma_{i j}}{z-\left(c_{i}+r_{i} \omega^{j}\right)}
$$

where $\gamma_{i j}$ is defined by

$$
\gamma_{i j}=\frac{1}{m} \sum_{l=0}^{m-1} \omega^{-j l} \sum_{z_{k} \in D_{i} \backslash U_{i-1}} q_{k} \cdot\left(\frac{z_{k}-c_{i}}{r_{i}}\right)^{l}, \quad \begin{aligned}
& i=1, \ldots, p \\
& j=0, \ldots, m-1
\end{aligned}
$$


with $U_{i}=\cup_{j \leq i} D_{j}$. The error of the approximation is bounded by

$$
\left|f(z)-g_{m}(z)\right| \leq \frac{2\left(a^{2}+1\right)|F(z)|}{\left(a^{m}-1\right)(a-1)^{2}}
$$

where

$$
F(z)=\sum_{j=1}^{n} \frac{\left|q_{j}\right|}{z-z_{j}}
$$

Lemma 3.5. Suppose that the discrete poles of Lemma 3.4 are replaced with a density $q$ defined on a curve $C$ with $C \subset U_{p}=D_{1} \cup \cdots \cup D_{p}$, specifically

$$
f(z)=\int_{C} \frac{q(\zeta)}{z-\zeta} d \zeta
$$

which is finite for $z$ outside $U_{p}$, and that $g_{m}$ is defined by (64) with $\gamma_{i j}$ defined by

$$
\gamma_{i j}=\frac{1}{m} \sum_{l=0}^{m-1} \omega^{-j l} \int_{C \cap\left(D_{i} \backslash U_{i-1}\right)} q(\zeta)\left(\frac{\zeta-c_{i}}{r_{i}}\right)^{l} d \zeta, \quad \begin{aligned}
& i=1, \ldots, p, \\
& j=0, \ldots, m-1,
\end{aligned}
$$

with $U_{i}=\cup_{j \leq i} D_{j}$. Then the bound (66) holds as before.

Lemma 3.3 enables us to approximate, with exponential convergence, a function defined as a sum of poles. The fundamental assumption is that the region of interest be "separated" from the pole locations. The notion of separation is effectively relaxed by covering the pole locations with disks of varying size in an adaptive manner. In Lemmas 3.4 and 3.5, we use this approach to derive our principal analytical result.

\section{Rational ApProximation of THE LogaRithmic DeRIVATIVE}

The logarithmic derivative of the Hankel function $G_{v}(z)$ approaches a constant as $z \rightarrow$ $\infty$ and is regular for finite $z \in \mathbb{C}$, except at $z=0$, which is a branch point, and at the zeros of $H_{v}^{(1)}(z)$, all simple. We can therefore develop a representation for $G_{v}$ analogous to that of the Mittag-Leffler theorem: the only addition is due to the branch cut on the negative imaginary axis. It will be convenient to work with $u_{v}(z)$, for which approximations to be introduced have simple error bounds.

Theorem 4.1. The function $u_{v}(z)=z G_{v}(z)$, where $G_{v}$ is defined for $v \in \mathbb{R}$ by (42) with the branch cut defined by (25), is given by the formula

$$
u_{v}(z)=i z-\frac{1}{2}+\sum_{n=1}^{N_{v}} \frac{h_{v, n}}{z-h_{v, n}}-\frac{1}{\pi i} \int_{0}^{\infty} \frac{\operatorname{Im}\left(u_{v}\left(r e^{-\pi i / 2}\right)\right)}{i r+z} d r
$$

for $z \in \mathbb{C}$ not in $\left\{0, h_{v, 1}, h_{v, 2}, \ldots, h_{v, N_{v}}\right\}$ and not on the negative imaginary axis. Here $h_{v, 1}, h_{v, 2}, \ldots, h_{v, N_{v}}$ denote the zeros of $H_{v}^{(1)}(z)$, which number $N_{v}$. 


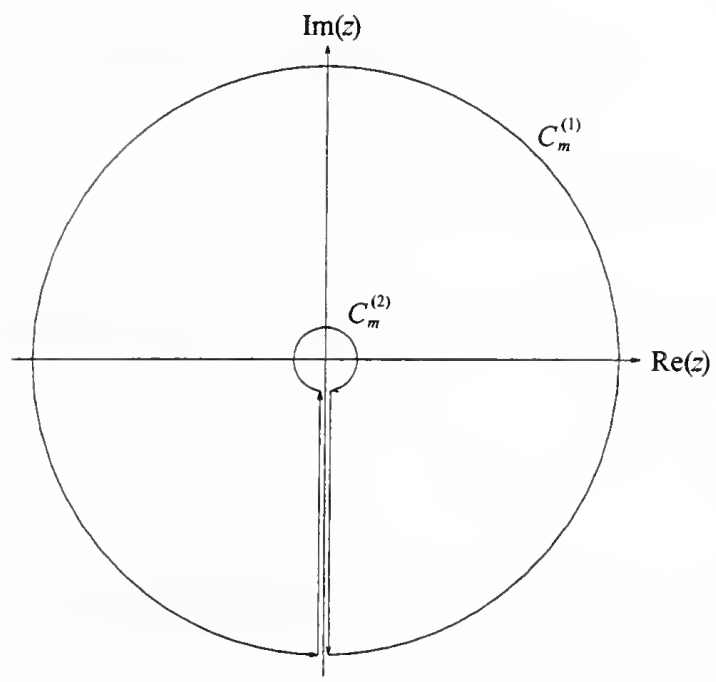

FIGURE 2. Integration contour $C_{m}$, with inner circle radius $1 / m$ and outer radius $m+1$.

Proof. The case of the spherical Hankel function, where $v=k+1 / 2$ for $k \in \mathbb{Z}$, is simple and we consider it first. Here $u_{\nu}(z)$ is a ratio of polynomials in $i z$ with real coefficients, which is clear from the observation that $u_{1 / 2}(z)=i z-1 / 2$ in combination with the recurrence (49). Hence

$$
u_{\nu}(z)=p(z)+\sum_{n=1}^{N_{v}} \frac{\alpha_{\nu, n}}{z-h_{v, n}},
$$

where $p$ is a polynomial and $\alpha_{v, n}$ is the residue of $u_{v}$ at $h_{v, n}$,

$$
\alpha_{\nu, n}=\lim _{z \rightarrow h_{v, n}}\left(z-h_{\nu, n}\right) u_{v}(z)=h_{\nu, n}
$$

by l'Hôpital's rule. We see from (46) that

$$
u_{v}(z) \sim i z-\frac{1}{2}+O\left(z^{-1}\right), \quad z \rightarrow \infty,
$$

whence

$$
p(z)=i z-\frac{1}{2} .
$$

Noting that $u_{v}(i y) \in \mathbb{R}$ for $y \in \mathbb{R}$, and combining (71), (72), and (74), we obtain (70).

We now consider the case $v \neq k+1 / 2, k \in \mathbb{Z}$, for which the origin is a branch point. For $m=1,2, \ldots$, we define $C_{m}$ to be the simple closed curve, shown in Figure 2, which proceeds counterclockwise along the circle $C_{m}^{(1)}$ of radius $m+1$ centered at the origin from $\arg z=-\pi / 2$ to $3 \pi / 2$, to the vertical segment $z=r e^{3 \pi i / 2}, r \in[1 / m, m+1]$, to the circle $C_{m}^{(2)}$ of radius $1 / m$ centered at the origin from $\arg z=3 \pi / 2$ to $-\pi / 2$, to the vertical 


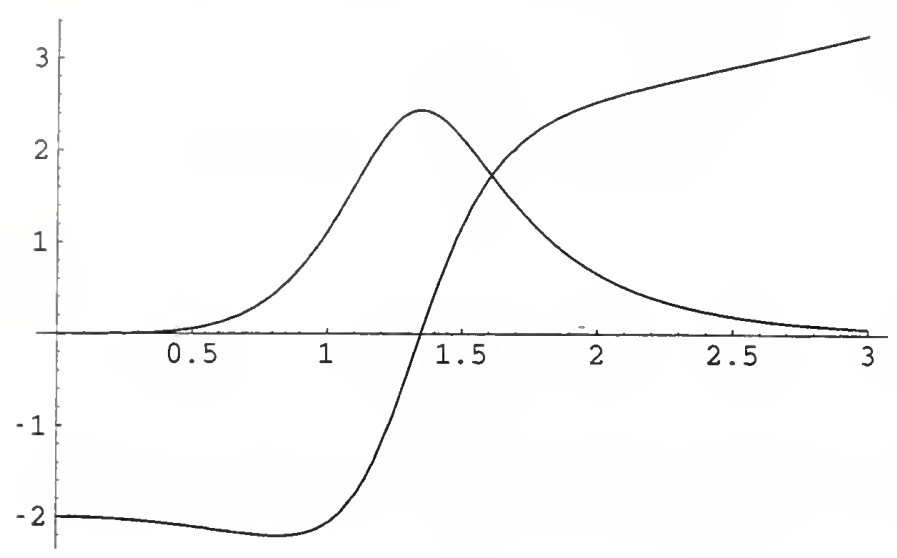

FIGURE 3. Plot of $\operatorname{Re}\left(u_{v}\left(r e^{-\pi i / 2}\right)\right)$, containing the zero crossing, and $\operatorname{Im}\left(u_{v}\left(r e^{-\pi i / 2}\right)\right)$, for $v=2$ and $r \in[0,3]$.

segment $z=r e^{-\pi i / 2}$, back to the first circle. Since none of the zeros of $H_{\nu}^{(1)}$ lies on the imaginary axis, $C_{m}$ encloses them all if $m$ is sufficiently large. For such $m$, and $z \in \mathbb{C}$ inside $C_{m}$ with $H_{v}^{(1)}(z) \neq 0$, the residue theorem gives

$$
\frac{1}{2 \pi i} \int_{C_{m}} \frac{u_{v}(\zeta)}{\zeta-z} d \zeta=u_{v}(z)+\sum_{n=1}^{N_{v}} \frac{h_{v, n}}{h_{v, n}-z}
$$

We now consider the separate pieces of the contour $C_{m}$. For the circles $C_{m}^{(1)}$ and $C_{m}^{(2)}$, we use the asymptotic expansion (73) about infinity and (45) about the origin to obtain

$$
\lim _{m \rightarrow \infty} \frac{1}{2 \pi i} \int_{C_{m}^{(1)}} \frac{u_{v}(\zeta)}{\zeta-z} d \zeta=i z-\frac{1}{2}, \quad \lim _{m \rightarrow \infty} \frac{1}{2 \pi i} \int_{C_{m}^{(2)}} \frac{u_{v}(\zeta)}{\zeta-z} d \zeta=0 .
$$

Now exploiting the symmetry $u_{v}\left(r e^{3 \pi i / 2}\right)=\overline{u_{v}\left(r e^{-\pi i / 2}\right)}$ from (44) for the vertical segments, we obtain

$$
\lim _{m \rightarrow \infty} \frac{1}{2 \pi i} \int_{C_{m}} \frac{u_{v}(\zeta)}{\zeta-z} d \zeta=i z-\frac{1}{2}+\frac{1}{2 \pi i} \int_{0}^{\infty} \frac{2 i \operatorname{Im}\left(u_{v}\left(r e^{-\pi i / 2}\right)\right)}{\left(r e^{-\pi i / 2}-z\right)} e^{-\pi i / 2} d r
$$

which, when combined with (75), yields (70) and the theorem.

The primary aim of this paper is to reduce the summation and integral of (70) to a similar summation involving dramatically fewer terms. To do so, we restrict $z$ to the upper halfplane and settle for an approximation. Such a representation is possible, for the poles of $u_{v}$ (zeros of $H_{v}^{(1)}$ ) lie entirely in the lower half-plane and do not cluster near the real axis. We first examine the behavior of $u_{\nu}$ on the negative imaginary axis.

The qualitative behavior of $u_{v}$ on the branch cut is illustrated by the case of $v=2$, shown in Figure 3. The plot changes little with changing $v$, except for the sign of $\operatorname{Im}\left(u_{v}(z)\right)$ and the sharpness of its extremum. 
Lemma 4.2. For $v \in \mathbb{R}, v \neq k+1 / 2, k \in \mathbb{Z}$, the function $u_{v}\left(r e^{-\pi i / 2}\right)$ is infinitely differentiable on $r \in(0, \infty)$ and has imaginary part satisfying the following formulae:

$$
\begin{aligned}
& \operatorname{Im}\left(u_{v}\left(r e^{-\pi i / 2}\right)\right)=\frac{\pi \cos (\nu \pi)}{\cos ^{2}(\nu \pi) K_{v}^{2}(r)+\left(\pi I_{\nu}(r)+\sin (\nu \pi) K_{v}(r)\right)^{2}} \neq 0,
\end{aligned}
$$

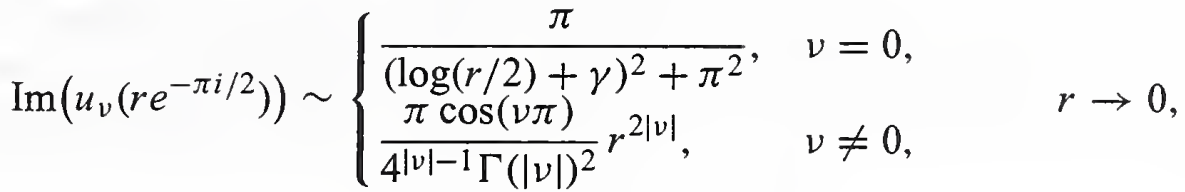

$$
\begin{aligned}
& \operatorname{Im}\left(u_{v}\left(r e^{-\pi i / 2}\right)\right) \sim 2 \cos (\nu \pi) r e^{-2 r} \text {, } \\
& r \rightarrow \infty, \\
& \operatorname{Im}\left(u_{v}\left(r e^{-\pi i / 2}\right)\right) \sim \frac{\cos (\nu \pi) \sqrt{r^{2}+\nu^{2}}}{\cosh (2 \nu \phi(r /|\nu|))+\sin (|\nu| \pi)}, \quad|\nu| \rightarrow \infty,
\end{aligned}
$$

where $\phi$ is defined in (41).

Proof. Infinite differentiability of $u_{v}(z)$ follows from the observation that $H_{v}^{(1)}(z) \neq 0$ on the negative imaginary axis. To derive (78) we recall (32) to obtain

$$
\operatorname{Im}\left(u_{v}\left(r e^{-\pi i / 2}\right)\right)=\frac{r \pi \cos (\nu \pi)\left(K_{v}(r) I_{v}^{\prime}(r)-K_{v}^{\prime}(r) I_{v}(r)\right)}{\cos ^{2}(\nu \pi) K_{v}^{2}(r)+\left(\pi I_{v}(r)+\sin (\nu \pi) K_{v}(r)\right)^{2}}
$$

then apply (31). The remaining formulas follow from the asymptotic forms of $K_{\nu}(r)$ and $I_{v}(r)$ for small and large $r$, and the uniform large $v$ expansions given in (33) through (36) and (40). Here we use the symmetry $u_{-v}=u_{v}$. Note that (79) is valid for $r /|v| \rightarrow 0$. The approximation (81) is nonuniform for $v \approx 2 k-1 / 2$ and $\pi I_{\nu}(r)+\sin (v \pi) K_{v}(r) \approx 0$.

Lemma 4.3. Given $\nu_{0}>0$ there exist constants $c_{0}$ and $c_{1}$ such that, for all $\nu \in \mathbb{R},|\nu| \geq \nu_{0}$, $v \neq k+1 / 2, k \in \mathbb{Z}$, and all $z$ satisfying $\operatorname{Im}(z) \geq 0$, the function

$$
f(z)=\int_{0}^{\infty} \frac{\operatorname{Im}\left(u_{v}\left(r e^{-\pi i / 2}\right)\right)}{i r+z} d r
$$

satisfies the bounds

$$
\frac{c_{0}}{1+|z| /|\nu|} \leq|f(z)| \leq \frac{c_{1}}{1+|z| /|\nu|} .
$$

Moreover, there exists $\delta>0$ such that for all $\nu \in \mathbb{R},|\nu| \geq \nu_{0}$, and $\varepsilon$ with $0<\varepsilon<1 / 2$, $f(z)$ admits an approximation $g(z)$ that is a sum of $d \leq \delta \cdot\left(1+|\nu|^{-1} \log (1 / \varepsilon)\right) \cdot \log (1 / \varepsilon)$ poles, with

$$
|f(z)-g(z)| \leq \varepsilon \cdot|f(z)|,
$$

provided $\operatorname{Im}(z) \geq 0$. 
Proof. We assume $v \neq k+1 / 2$ for integral $k$ and begin by changing variables, $r=|v| w$, so that

$$
f(z)=\int_{0}^{\infty} \frac{\operatorname{Im}\left(u_{v}\left(|v| w e^{-\pi i / 2}\right)\right)}{i w+z /|\nu|} d w=\int_{0}^{\infty} \mu_{z}(w) d w .
$$

From the nonvanishing of $\mu_{z}$ and its asymptotic behavior in $w$, it is clear that (84) holds for $|v| \in\left(\nu_{0}, \nu_{1}\right)$ and any fixed $\nu_{1}>v_{0}$. Using (81) for $|v|$ large but bounded away from $2 k-1 / 2$ for integral $k$, an application of Watson's lemma to (83) focuses on the unique positive zero, $w^{*}$, of $\phi$ defined in (41). As the derivative of this function is positive, we conclude

$$
f(z) \sim \frac{\alpha \cos (\nu \pi)}{i w^{*}+z /|\nu|},
$$

where $\alpha$ is a function of $w^{*}$, so that (84) clearly holds. However, as $v \rightarrow 2 k-1 / 2$, the denominator on the right-hand side of (81) may nearly vanish at $w^{*}$ and the expansion loses its uniformity. Setting $\cos (\nu \pi)=\eta$ in these cases, we see that the denominator has a minimum which is bounded below by $O\left(\eta^{2}\right)$. Hence in an $O\left(|\nu|^{-1}\right)$ neighborhood of the minimum which includes $w^{*}$ we have

$$
\int \mu_{z}(w) \approx \frac{\eta|\nu| \sqrt{1+\left(w^{*}\right)^{2}}}{i w^{*}+z /|v|} \int_{-\gamma /|\nu|}^{\gamma /|\nu|} \frac{1}{\eta^{2}+\beta^{2} \nu^{2} s^{2}} d s
$$

which by the change of variables $s=\eta z /|\nu|$ is seen to satisfy the upper bound in (84) uniformly in $\eta$. As the rest of the integral is small, the upper bound holds.

We now move on to the approximation. For a positive integer $m$ and a positive number $w_{0}$, we define intervals $I_{0}=\left(0, w_{0}\right), I_{j}=\left(2^{j-1} w_{0}, 2^{j} w_{0}\right)$ for $j=1, \ldots, m$, and $I_{m+1}=$ $\left(2^{m} w_{0}, \infty\right)$. Now

$$
f(z)=f_{0}(z)+f_{1}(z)+f_{2}(z)
$$

where $f_{0}, f_{1}$, and $f_{2}$ are defined by the formulae

$$
f_{0}(z)=\int_{I_{0}} \mu_{z}(w) d w, \quad f_{1}(z)=\sum_{j=1}^{m} \int_{I_{j}} \mu_{z}(w) d w, \quad f_{2}(z)=\int_{I_{m+1}} \mu_{z}(w) d w .
$$

We will now choose $w_{0}$ and $m$ so that $f_{0}$ and $f_{2}$ can be ignored and then use Lemma 3.5 to approximate $f_{1}$. Using (79) and (81) and taking $w_{0}$ sufficiently small we have, for some constant $c_{2}$ independent of $v$,

$$
\left|f_{0}(z)\right| \leq \frac{c_{2}|\nu|}{1+|z| /|\nu|}\left(\frac{3 e}{4}\right)^{2|\nu|} \int_{0}^{w_{0}} w^{2|\nu|-1} d w \leq \frac{c_{2}}{1+|z| /|\nu|}\left(\frac{3 e}{4} w_{0}\right)^{2|\nu|} .
$$

Hence, a choice of

$$
w_{0}=O\left(\varepsilon^{1 /(2|v|)}\right), \quad \varepsilon \rightarrow 0,
$$


suffices to guarantee

$$
\left|f_{0}(z)\right| \leq \frac{\varepsilon}{3}|f(z)|
$$

in the closed upper half-plane. Now using (80) and (81) and assuming $m$ sufficiently large we have, for some constant $c_{3}$ independent of $v$,

$$
\left|f_{2}(z)\right| \leq \frac{c_{3}|\nu|}{1+|z| /|\nu|} \int_{2^{m} w_{0}}^{\infty} w e^{-|\nu| w} d w \leq \frac{c_{2}}{1+|z| /|\nu|} 2^{m} w_{0} e^{-|\nu| 2^{m} w_{0}}
$$

From (92), choosing

$$
m \geq m_{0}+m_{1} \frac{1}{|\nu|} \log \frac{1}{\varepsilon},
$$

for appropriate $m_{0}$ and $m_{1}$ independent of $\nu$ and $\varepsilon$ leads to

$$
\left|f_{2}(z)\right| \leq \frac{\varepsilon}{3}|f(z)|
$$

Finally, we apply Lemma 3.5 to the approximation of $f_{1}$ The error involves the function $F_{1}=\int\left|\operatorname{Im}\left(u_{v}\right)\right| /(i r+z) d r$, but we note that $\left|F_{1}\right|=\left|f_{1}\right|$. Using $p$ poles for each $j$ we produce a $p \cdot m$-pole approximation $g(z)$ with an error estimate, again for $\operatorname{Im}(z) \geq 0$, given by

$$
\left|f_{1}(z)-g(z)\right| \leq \frac{5}{3^{p}-1}\left|f_{1}(z)\right|
$$

A choice of

$$
p=O\left(\log \frac{1}{\varepsilon}\right)
$$

enforces

$$
\left|f_{1}(z)-g(z)\right| \leq \frac{\varepsilon}{3}|f(z)|
$$

By combining (93), (96), (99), and the triangle inequality, we obtain (85) with the number of poles, $d=p \cdot m$, satisfying the stated bound.

The case $v=0$ requires special treatment. First, the direct application of the preceding arguments leads to a significantly larger upper bound on the number of poles. Secondly, we note that $u_{0}(0)=0$, so that relative error bounds near $z=0$ require a vanishing absolute error. Finally, the lack of regularity of $u_{0}(z)$ at $z=0$ precludes uniform rational approximation, as discussed in [9]. Therefore, we relax the condition $\operatorname{Im}(z) \geq 0$ to $\operatorname{Im}(z) \geq$ $\eta>0$. By (20) this will lead to good approximate convolutions for times $T \leq \eta^{-1}$.

Lemma 4.4. There exists $\delta>0$ such that for all $\varepsilon, 0<\varepsilon<1 / 2$ and $\eta, 0<\eta<1 / 2$, the function $f(z)=u_{0}(z)-i z+1 / 2$ admits an approximation $g(z)$ that is a sum of $d \leq \delta \cdot(\log (1 / \eta)+\log \log (1 / \varepsilon)) \cdot \log (1 / \varepsilon)$ poles, with

$$
|f(z)-g(z)| \leq \varepsilon \cdot|f(z)|,
$$

provided $\operatorname{Im}(z) \geq \eta$. 
Proof. Note that since $u_{0}(z)$ has no poles, $f(z)$ is given by (83), and satisfies (84). Define intervals

$$
I_{j}=\left(\left(2^{j-1}-1\right) \eta,\left(2^{j}-1\right) \eta\right) \text { for } j=1, \ldots, m, \quad I_{m+1}=\left(\left(2^{m}-1\right) \eta, \infty\right) .
$$

Now

$$
f(z)=f_{1}(z)+f_{2}(z)
$$

where $f_{1}$, and $f_{2}$ are defined by the formulae

$$
f_{1}(z)=\sum_{j=1}^{m} \int_{I_{j}} \frac{\operatorname{Im}\left(u_{0}\left(r e^{-\pi i / 2}\right)\right)}{i r+z} d r, \quad f_{2}(z)=\int_{I_{m+1}} \frac{\operatorname{Im}\left(u_{0}\left(r e^{-\pi i / 2}\right)\right)}{i r+z} d r .
$$

We will now choose $m$ so that $f_{2}$ can be ignored and then use Lemma 3.5 to approximate $f_{1}$. Using (80) and assuming $m$ sufficiently large we have, for some constant $c$,

$$
\left|f_{2}(z)\right| \leq \frac{c}{1+|z|} \int_{\left(2^{m}-1\right) \eta}^{\infty} r e^{-2 r} d w \leq \frac{c}{1+|z|} 2^{m-1} \eta e^{-2^{m} \eta} .
$$

Hence, choosing

$$
m \geq m_{0}(\log (1 / \eta)+\log \log (1 / \varepsilon)),
$$

for appropriate $m_{0}$ independent of $\eta$ and $\varepsilon$ leads to

$$
\left|f_{2}(z)\right| \leq \frac{\varepsilon}{2}|f(z)|
$$

Finally, we apply Lemma 3.5 to the approximation of $f_{1}$. Using $p$ poles for each $j$ we produce a $p \cdot m$-pole approximation $g(z)$ with an error estimate for $\operatorname{Im}(z) \geq \eta$ given by

$$
\left|f_{1}(z)-g(z)\right| \leq \frac{5}{3^{p}-1}\left|f_{1}(z)\right| .
$$

A choice of

$$
p=O\left(\log \frac{1}{\varepsilon}\right)
$$

enforces

$$
\left|f_{1}(z)-g(z)\right| \leq \frac{\varepsilon}{2}|f(z)| .
$$

By (105), (108), and the triangle inequality, (100) is achieved with the number of poles, $d=p \cdot m$, satisfying the stated bound.

We now consider the contribution of the poles.

Lemma 4.5. There exist constants $C_{0}, C_{1}, \delta>0$ such that for all $v, \varepsilon \in \mathbb{R}$ with $2 \leq|v|$ and $0<\varepsilon<1 / 2$ the function

$$
h(z)=\sum_{n=1}^{N_{v}} \frac{h_{v, n}}{z-h_{v, n}},
$$


where $h_{v, 1}, \ldots, h_{v, N_{v}}$ are the roots of $H_{v}^{(1)}$, satisfies the inequalities

$$
\frac{C_{1}|\nu|}{1+|z| /|\nu|} \leq|h(z)| \leq \frac{C_{2}|\nu|}{1+|z| /|\nu|},
$$

and admits an approximation $g(z)$ that is a sum of $d \leq \delta \cdot \log |\nu| \cdot \log (1 / \varepsilon)$ poles, with

$$
|h(z)-g(z)| \leq \varepsilon \cdot|h(z)|
$$

provided $\operatorname{Im}(z) \geq 0$.

Proof. The curve $C$ defined in Lemma 3.2, near which $h_{v, 1} /|\nu|, \ldots, h_{v, N_{v}} /|\nu|$ lie, is contained in disks separated from the real axis. If we denote the disk of radius $r$ centered at $c$ by $D(r, c)$, then the disks

$$
\left\{D(-\operatorname{Im}(z), z)|z \in C,| \arg z-\pi / 2 \mid=\pi / 2+\pi / 2^{n}, n=1,2, \ldots\right\},
$$

for example, contain $C \backslash\{+1,-1\}$. From (52), the root $h_{v, 1}$ closest to the real axis satisfies

$$
\arg h_{v, 1} \sim \frac{a_{1} \sqrt{3}}{2^{4 / 3}}|v|^{-2 / 3},
$$

hence is contained in a disk of (112) with $n \approx \log _{2}\left(2^{4 / 3} 3^{-1 / 2} \pi\left(-a_{1}\right)^{-1}|\nu|^{2 / 3}\right)$, and all of the roots are contained in $O(\log |\nu|)$ of the disks. Now applying Lemma 3.4 we obtain (111) with $|h|$ replaced by $|H|=\left|\sum\right| h_{v, n}\left|/\left(z-h_{v, n}\right)\right|$. To obtain the upper bound in (110) for both $h$ and $H$ we note first that it is trivial except for $|z / \nu| \approx 1$. A detailed analysis of the roots as described by Lemma 3.2 shows that

$$
\left|\operatorname{Im}\left(h_{v, j}\right)\right| \geq c j^{2 / 3}|\nu|^{1 / 3}
$$

Hence, for $|z / \nu| \approx 1$,

$$
\sum_{j}\left|\frac{h_{\nu, j}}{z-h_{v, j}}\right| \leq C|\nu|^{2 / 3} \sum_{j=1}^{|\nu|} j^{-2 / 3} \leq 3 C|\nu| .
$$

The lower bound in (110) is again obvious except for $|z / \nu| \approx 1$. Then, however, we note that

$$
h(z)=u_{v}(z)-i z+1 / 2-f(z) .
$$

Since, from (47), $\left|u_{v}(z)\right|=O\left(|v|^{2 / 3}\right)$ for $|z / v| \approx 1$ and $|f(z)|=O(1)$ by (84) the righthand side is dominated by $-i z$ and $|h(z)|=O(|\nu|)$.

The combination of Theorem 4.1 and Lemmas 4.3 and 4.5 suffices to prove our principal analytical result.

Theorem 4.6. Given $v_{0}>0$ there exists $\delta>0$ such that for all $v \in \mathbb{R},|v| \geq v_{0}$, and $0<\varepsilon<1 / 2$ there exists $d$ with

$$
d \leq \delta\left(\log |\nu| \cdot \log (1 / \varepsilon)+\log ^{2}|\nu|+|\nu|^{-1} \log ^{2}(1 / \varepsilon)\right)
$$


and complex numbers $\alpha_{1}, \ldots, \alpha_{d}$ and $\beta_{1}, \ldots, \beta_{d}$, depending on $v$ and $\varepsilon$, such that the function

$$
U_{\nu, \varepsilon}(z)=i z-\frac{1}{2}+\sum_{n=1}^{d} \frac{\alpha_{n}}{z-\beta_{n}}
$$

approximates $u_{v}(z)$ with the bound

$$
\left|u_{v}(z)-U_{v, \varepsilon}(z)\right| \leq \varepsilon \cdot\left|u_{v}(z)\right|,
$$

provided that $\operatorname{Im}(z) \geq 0$. Furthermore

$$
\left(\int_{-\infty}^{\infty}\left|u_{v}(x)-U_{v, \varepsilon}(x)\right|^{2} d x\right)^{1 / 2} \leq \varepsilon \cdot\left(\int_{-\infty}^{\infty}\left|u_{v}(x)-i x+1 / 2\right|^{2} d x\right)^{1 / 2}
$$

Proof. We first note the lower bound

$$
\left|u_{v}(z)-i z+1 / 2\right| \geq \frac{c|v|}{1+|z| /|v|}
$$

For $v>0$ the function is nonvanishing and has the correct asymptotic behavior, so we need only consider the case of $|\nu|$ large. The result then follows from (47). This proves (120) and (119) with $u_{v}$ replaced by $u_{v}-i z+1 / 2$ on the right-hand side. From (47) we have

$$
\left|u_{v}(z)-i z+1 / 2\right| \leq c|v|^{1 / 3}\left|u_{\nu}(z)\right|
$$

so that the final result follows from the scaling $\varepsilon \rightarrow|\nu|^{-1 / 3} \varepsilon$.

The number of poles in (117) required to approximate $u_{v}(z)$ to a tolerance $\varepsilon$, depends on both $\varepsilon$ and $\nu$. The asymptotic dependence on $\varepsilon$ is proportional to $|\nu|^{-1} \log ^{2}(1 / \varepsilon)$. We will see in the numerical examples, however, that this term is important only for small $|\nu|$; otherwise the dominant term is the first, for an asymptotic dependence of $O(\log |\nu| \cdot \log (1 / \varepsilon))$. As we generally have $\varepsilon \ll|\nu|^{-1}$ in practice, the term $\log ^{2}|\nu|$ is of less importance.

Similarly, Lemma 4.4 leads to the following theorem for $\nu=0$.

Theorem 4.7. There exists $\delta>0$ such that for all $\varepsilon, 0<\varepsilon<1 / 2$ and $\eta, 0<\eta<1 / 2$ there exists $d \leq \delta \cdot(\log (1 / \eta) \cdot \log (1 / \varepsilon)+\log \log (1 / \varepsilon)+\log \log (1 / \eta))$ and complex numbers $\alpha_{1}, \ldots, \alpha_{d}$ and $\beta_{1}, \ldots, \beta_{d}$, depending on $\eta$ and $\varepsilon$, such that the function

$$
U_{0, \varepsilon}(z)=i z-\frac{1}{2}+\sum_{n=1}^{d} \frac{\alpha_{n}}{z-\beta_{n}}
$$

approximates $u_{0}(z)$ with the bound

$$
\left|u_{0}(z)-U_{0, \varepsilon}(z)\right| \leq \varepsilon \cdot\left|u_{0}(z)\right|
$$


provided that $\operatorname{Im}(z) \geq \eta$. Furthermore

$$
\begin{aligned}
& \left(\int_{-\infty}^{\infty}\left|u_{0}(x+i \eta)-U_{0, \varepsilon}(x+i \eta)\right|^{2} d x\right)^{1 / 2} \\
& \quad \leq \varepsilon \cdot\left(\int_{-\infty}^{\infty}\left|u_{v}(x+i \eta)-i x+\eta+1 / 2\right|^{2} d x\right)^{1 / 2}
\end{aligned}
$$

Proof. Again we already have (124) with $u_{0}(z)-i z+1 / 2$ on the right-hand side. By (45) we find

$$
\left|u_{0}(z)-i z+1 / 2\right| \leq c \log (1 / \eta)\left|u_{0}(z)\right| \text {. }
$$

The theorem follows from the scaling $\varepsilon \rightarrow \log ^{-1}(1 / \eta) \varepsilon$.

As we must take $\eta=T^{-1}$, we see that the number of poles required may grow like $\log (1 / \varepsilon) \cdot \log T+\log T \cdot \log \log T$. However, this is only for the mode $n=0$ in the two dimensional case. In short, the $T$ dependence is insignificant in practice.

\section{Computation of the Rational REPREsentations}

Analytical error bound estimates developed in the previous sections are based on maximum norm errors as in (19) and (20). In numerical computation it is often convenient, however, to obtain least squares solutions. Our method of computing a rational function $U_{v, \varepsilon}$ that satisfies (119) is to enforce (120). An alternative approach would be to use rational Chebyshev approximation as developed by Trefethen and Gutknecht [22, 23, 24].

In the numerical computations, we work with

$$
\tilde{u}_{v}(z)=u_{v}(z)-i z+1 / 2
$$

and its sum-of-poles approximation $\tilde{U}_{\nu, \varepsilon}(z)=U_{v, \varepsilon}(z)-i z+1 / 2$. In particular, we have the nonlinear least squares problem

$$
\min _{P, Q} \int_{-\infty}^{\infty}\left|\frac{P(x)}{Q(x)}-\tilde{u}_{v}(x)\right|^{2} d x
$$

for $P, Q$ polynomials with $\operatorname{deg}(P)+1=\operatorname{deg}(Q)=d$. Problem (128) is not only nonlinear, but also very poorly conditioned when $P, Q$ are represented in terms of their monomial coefficients. We apply two tactics for coping with these difficulties: linearization and orthogonalization.

We linearize the problem by starting with a good estimate of $Q$, and updating $P, Q$ iteratively. In particular, we solve the linear least squares problem

$$
\min _{P^{(i+1)}, Q^{(i+1)}} \int_{-\infty}^{\infty}\left|\frac{P^{(i+1)}(x)}{Q^{(i)}(x)}-\frac{Q^{(i+1)}(x)}{Q^{(i)}(x)} \tilde{u}_{v}(x)\right|^{2} d x,
$$

where the integral is replaced by a quadrature. The initial values $P^{(0)}, Q^{(0)}$ are obtained by exploiting the asymptotic expansion (46) and the recurrence (49). We find that two to three iterations of (129) generally suffice. 
The quadrature for (129) is derived by first changing variables,

$$
\int_{-\infty}^{\infty} f(x) d x=\int_{-\pi / 2}^{\pi / 2} f(\tan \theta) \sec ^{2} \theta d \theta \approx \sum_{i=1}^{m} w_{i} f\left(\tan \theta_{i}\right) \sec ^{2} \theta_{i},
$$

where $\theta_{1}, \ldots, \theta_{m}$ and $w_{1} \ldots, w_{m}$ denote appropriate quadrature nodes and weights. The transformed integrand is periodic on the interval $[-\pi / 2, \pi / 2]$, so the trapezoidal rule (or midpoint rule) is an obvious candidate. The integrand is infinitely continously differentiable, except at $\theta=0$, where its regularity is of order $2|v|$. For $|v|>8$ (say), the trapezoidal rule delivers at least 16th-order convergence and is very effective. For small $|v|$, however, a quadrature that adjusts for the complicated singularity at $\theta=0$ is needed. Here we can successively subdivide the interval near the singularity, applying high-order quadratures on each subinterval (see, for example, [25]). We, instead, apply quadratures designed to handle a variety of singularities efficiently [26].

The quadrature discretization of (129) cannot be solved as a least-squares problem by standard techniques, due to its extremely poor conditioning. We avoid forming the corresponding matrix; rather we solve the least squares problem by Gram-Schmidt orthogonalization. The $2 d+1$ functions

$$
\tilde{u}_{\nu}, 1, x \tilde{u}_{v}, x, \ldots, x^{d-1} \tilde{u}_{\nu}, x^{d-1}, x^{d} \tilde{u}_{\nu}
$$

are orthogonalized under the real inner product

$$
\langle f, g\rangle_{i}=\int_{-\infty}^{\infty} \frac{\operatorname{Re}(f(x) \bar{g}(x))}{\left|Q^{(i)}(x)\right|^{2}} d x
$$

to obtain the orthogonal functions

$$
g_{n}(x)= \begin{cases}\tilde{u}_{\nu}(x), & n=1, \\ 1, & n=2, \\ x g_{n-2}(x)-\sum_{j=1}^{\min \{4, n-1\}} c_{n j} g_{n-j}(x), & n=3, \ldots, 2 d+1,\end{cases}
$$

where

$$
c_{n j}=\frac{\left\langle x g_{n-2}, g_{n-j}\right\rangle_{i}}{\left\langle g_{n-j}, g_{n-j}\right\rangle_{i}}, \quad \begin{aligned}
& n=3, \ldots, 2 d+1, \\
& j=1, \ldots, \min \{4, n-1\}
\end{aligned}
$$

Now

$$
g_{2 d+1}=-P^{(i+1)}+\tilde{u}_{\nu} Q^{(i+1)},
$$

so $P^{(i+1)}$ and $Q^{(i+1)}$ are computed from the recurrence coefficients $c_{n j}$ by splitting (133) into even and odd-numbered parts.

For some applications, including nonreflecting boundary kernels, it is convenient to represent $P / Q$ as a sum of poles,

$$
\frac{P(z)}{Q(z)}=\sum_{n=1}^{d} \frac{\alpha_{n}}{z-\beta_{n}}
$$


We compute $\beta_{1}, \ldots, \beta_{d}$ (zeros of $Q$ ) by Newton iteration with zero suppression (see, for example, [27]) by the formula

$$
\beta_{n}^{(j+1)}=\beta_{n}^{(j)}-\frac{Q\left(\beta_{n}^{(j)}\right)}{Q^{\prime}\left(\beta_{n}^{(j)}\right)-\sum_{k=1}^{n-1} \frac{Q\left(\beta_{n}^{(j)}\right)}{\beta_{n}^{(j)}-\beta_{k}}},
$$

where $\beta_{1}, \ldots, \beta_{n-1}$ are the previously computed zeros of $Q$. Then $\alpha_{1}, \ldots, \alpha_{d}$ are computed by the formula $\alpha_{n}=P\left(\beta_{n}\right) / Q^{\prime}\left(\beta_{n}\right)$. The derivative $Q^{\prime}(z)$ is obtained by differentiating the recurrence (133).

\section{Numerical Results}

We have implemented the algorithm described in Section 5 to compute the representations of $\sigma_{n}$ and $\omega_{n}$ through their Laplace transforms. Recall that for the cylinder kernels, $\sigma_{n}$, we have $v=n$ while for the sphere kernels, $\omega_{n}$, we have $\nu=n+1 / 2$. Table 1 presents the sizes of the representations for $\varepsilon=10^{-6}, 10^{-8}$, and $10^{-15}$ in (120). For the cylinder kernels, which are affected by the branch cut, the number of poles for small $n$ is higher than for the sphere kernels. This discrepancy, however, rapidly vanishes as $n$ increases and the asymptotic performance ensues. The $\log (1 / \varepsilon)$ dependence of the number of poles for $n \geq 10$ is clear.

For $\varepsilon=10^{-8}$ we have also computed the maximum norm relative errors which appear in (19) by sampling on a fine mesh. For the cylinder kernel with $n=0$, we expect an $O(1)$ error in a small interval about the origin due to (79). However, errors of less than $\varepsilon$ are achieved for $|s|>5 \times 10^{-7}$. This implies a similar accuracy in the approximation of the convolution for times of order $10^{6}$. For all other cases the maximum norm relative errors are of order $\varepsilon$.

Finally, Table 2 presents poles and coefficients for the cylinder kernels for $n=1, \ldots, 4$ and $\varepsilon=10^{-6}$, to allow comparison by a reader interested in repeating our calculations. Note that the pole locations are written in terms of $s=z / i$. Extensive tables will be made available on the Web at http://math.nist.gov/mcsd/Staff/BAlpert.

Remark. Our approximate representation of the nonreflecting boundary kernel could be used to reduce the cost of the method introduced by Grote and Keller [7]. The differential operators of degree $n$ obtained in their derivation need only be replaced by the corresponding differential operators of degree $\log n$ for any specified accuracy. It is interesting to note that in the two-dimensional case, where the approach of [7] does not apply, the analysis described above can be used to derive an integrodifferential formulation in the same spirit.

\section{SUMMARY}

In this paper we have introduced new representations for the logarithmic derivative of a Hankel function of real order, that scale in size as the logarithm of the order. An algorithm to compute the representations was presented and our numerical results demonstrate that 
TABLE 1. Number $d$ of poles to represent the Laplace transform of nonreflecting boundary kernels $\sigma_{n}$ and $\omega_{n}$, for various values of $\varepsilon$.

\begin{tabular}{|c|c|c|c|c|c|c|c|}
\hline \multicolumn{4}{|c|}{$\varepsilon=10^{-6}$} & & & & \\
\hline \multicolumn{2}{|l|}{$\sigma_{n}$} & \multicolumn{2}{|l|}{$\omega_{n}$} & & & & \\
\hline$n$ & $d$ & \multirow[t]{2}{*}{$n$} & $d$ & \multirow{2}{*}{\multicolumn{4}{|c|}{$\varepsilon=10^{-15}$}} \\
\hline \multicolumn{2}{|c|}{026} & & & & & \multirow{2}{*}{\multicolumn{2}{|c|}{$\omega_{n}$}} \\
\hline 1 & 9 & & & \multicolumn{2}{|l|}{$\sigma_{n}$} & & \\
\hline 2 & 6 & \multirow[b]{2}{*}{$0-5$} & \multirow[b]{2}{*}{$n$} & \multicolumn{2}{|c|}{$\frac{d}{11}$} & $n$ & $d$ \\
\hline $3-6$ & 5 & & & 1 & 41 & & \\
\hline $7-8$ & 6 & $6-8$ & 6 & 2 & 24 & & \\
\hline $9-12$ & 7 & $9-12$ & 7 & 3 & 18 & & \\
\hline $13-19$ & 8 & $13-19$ & 8 & 4 & 15 & & \\
\hline $20-31$ & 9 & $20-31$ & 9 & 5 & 14 & & \\
\hline $32-51$ & 10 & $32-51$ & 10 & 6 & 13 & - & \\
\hline $52-86$ & 11 & $52-86$ & 11 & $7-12$ & 12 & & \\
\hline $87-147$ & 12 & $87-147$ & 12 & $13-14$ & 13 & $0-13$ & $n$ \\
\hline $148-227$ & 13 & $148-228$ & 13 & $15-16$ & 14 & $14-15$ & 14 \\
\hline $228-401$ & 14 & $229-402$ & 14 & $17-18$ & 15 & $16-18$ & 15 \\
\hline $402-728$ & 15 & $403-728$ & 15 & $19-22$ & 16 & $19-21$ & 16 \\
\hline $729-1024$ & 16 & $729-1024$ & 16 & $23-26$ & 17 & $22-25$ & 17 \\
\hline \multicolumn{4}{|c|}{$\varepsilon=10^{-8}$} & $27-31$ & 18 & $26-30$ & 18 \\
\hline \multicolumn{2}{|l|}{$\sigma_{n}$} & \multicolumn{2}{|l|}{$\omega_{n}$} & $38-45$ & $\begin{array}{l}19 \\
20\end{array}$ & $\begin{array}{l}31-36 \\
37-44\end{array}$ & $\begin{array}{l}19 \\
20\end{array}$ \\
\hline$n$ & $d$ & $n$ & $d$ & $46-54$ & 21 & $45-53$ & 21 \\
\hline 0 & 44 & & & $55-65$ & 22 & $54-65$ & 22 \\
\hline 1 & 15 & & & $66-79$ & 23 & $66-79$ & 23 \\
\hline 2 & 9 & & & $80-97$ & 24 & $80-96$ & 24 \\
\hline $3-8$ & 7 & $0-7$ & $n$ & $98-118$ & 25 & $97-118$ & 25 \\
\hline $9-10$ & 8 & $8-10$ & 8 & $119-145$ & 26 & $119-144$ & 26 \\
\hline $11-14$ & 9 & $11-14$ & 9 & $146-177$ & 27 & $145-176$ & 27 \\
\hline $15-20$ & 10 & $15-19$ & 10 & $178-216$ & 28 & $177-216$ & 28 \\
\hline $21-28$ & 11 & $20-28$ & 11 & $217-265$ & 29 & $217-264$ & 29 \\
\hline $29-41$ & 12 & $29-40$ & 12 & $266-324$ & 30 & $265-324$ & 30 \\
\hline $42-58$ & 13 & $41-57$ & 13 & $325-397$ & 31 & $325-396$ & 31 \\
\hline $59-84$ & 14 & $58-83$ & 14 & $398-486$ & 32 & $397-485$ & 32 \\
\hline $85-123$ & 15 & $84-123$ & 15 & $487-595$ & 33 & $486-594$ & 33 \\
\hline $124-183$ & 16 & $124-183$ & 16 & $596-728$ & 34 & $595-727$ & 34 \\
\hline $184-275$ & 17 & $184-275$ & 17 & $729-890$ & 35 & $728-890$ & 35 \\
\hline $276-418$ & 18 & $276-418$ & 18 & $891-1024$ & 36 & $891-1024$ & 36 \\
\hline $419-638$ & 19 & $419-637$ & 19 & & & & \\
\hline $639-971$ & 20 & $638-971$ & 20 & & & & \\
\hline $972-1024$ & 21 & $972-1024$ & 21 & & & & \\
\hline
\end{tabular}

the new representations are modest in size for orders and accuracies likely to be of practical interest. 
TABLE 2. Laplace transform of cylinder kernel $\sigma_{n}$ defined in (13), approximated as a sum of $d$ poles, for $n=1, \ldots, 4$ and $\varepsilon=10^{-6}$.

\begin{tabular}{|c|c|c|c|c|}
\hline \multirow[b]{2}{*}{$n \quad d$} & \multicolumn{2}{|c|}{ Pole Coefficient } & \multicolumn{2}{|c|}{ Pole Location } \\
\hline & $\mathrm{Re}$ & Im & $\operatorname{Re}$ & $\operatorname{Im}$ \\
\hline \multirow[t]{9}{*}{19} & $-0.426478 E-02$ & $0.000000 E+00$ & $-0.368403 E+01$ & $0.000000 E+00$ \\
\hline & $-0.416255 E-01$ & $0.000000 E+00$ & $-0.205860 E+01$ & $0.000000 E+00$ \\
\hline & $-0.122665 E+00$ & $0.000000 E+00$ & $-0.118994 E+01$ & $0.000000 E+00$ \\
\hline & $-0.143704 E+00$ & $0.000000 E+00$ & $-0.717570 E+00$ & $0.000000 E+00$ \\
\hline & $-0.530662 E-01$ & $0.000000 E+00$ & $-0.423506 E+00$ & $0.000000 E+00$ \\
\hline & $-0.863872 E-02$ & $0.000000 E+00$ & $-0.223111 E+00$ & $0.000000 E+00$ \\
\hline & $-0.961472 E-03$ & $0.000000 E+00$ & $-0.103710 E+00$ & $0.000000 E+00$ \\
\hline & $-0.721548 E-04$ & $0.000000 E+00$ & $-0.409342 E-01$ & $0.000000 E+00$ \\
\hline & $-0.250102 E-05$ & $0.000000 E+00$ & $-0.117156 E-01$ & $0.000000 E+00$ \\
\hline \multirow[t]{6}{*}{26} & $0.218164 E-01$ & $0.000000 E+00$ & $-0.333263 E+01$ & $0.000000 E+00$ \\
\hline & $0.860648 E+00$ & $0.000000 E+00$ & $-0.162945 E+01$ & $0.000000 E+00$ \\
\hline & $-0.138934 E+01$ & $0.162069 E+00$ & $-0.125843 E+01$ & $0.412637 E+00$ \\
\hline & $-0.138934 E+01$ & $-0.162069 E+00$ & $-0.125843 E+01$ & $-0.412637 E+00$ \\
\hline & $0.209905 E-01$ & $0.000000 E+00$ & $-0.612710 E+00$ & $0.000000 E+00$ \\
\hline & $0.232032 E-03$ & $0.000000 E+00$ & $-0.240327 E+00$ & $0.000000 E+00$ \\
\hline \multirow[t]{5}{*}{35} & $-0.179277 E+00$ & $0.000000 E+00$ & $-0.309775 E+01$ & $0.000000 E+00$ \\
\hline & $-0.168335 E+01$ & $0.129111 E+01$ & $-0.167998 E+01$ & $0.130784 E+01$ \\
\hline & $-0.168335 E+01$ & $-0.129111 E+01$ & $-0.167998 E+01$ & $-0.130784 E+01$ \\
\hline & $-0.816322 E+00$ & $0.000000 E+00$ & $-0.187260 E+01$ & $0.000000 E+00$ \\
\hline & $-0.126962 E-01$ & $0.000000 E+00$ & $-0.950854 E+00$ & $0.000000 E+00$ \\
\hline \multirow[t]{5}{*}{$\begin{array}{ll}45 \\
\end{array}$} & $-0.197725 E+01$ & $0.220886 E+01$ & $-0.197861 E+01$ & $0.220444 E+01$ \\
\hline & $-0.197725 E+01$ & $-0.220886 E+01$ & $-0.197861 E+01$ & $-0.220444 E+01$ \\
\hline & $-0.219247 E+01$ & $0.216535 E+01$ & $-0.282304 E+01$ & $0.382237 E+00$ \\
\hline & $-0.219247 E+01$ & $-0.216535 E+01$ & $-0.282304 E+01$ & $-0.382237 E+00$ \\
\hline & $0.464435 E+00$ & $0.000000 E+00$ & $-0.201159 E+01$ & $0.000000 E+00$ \\
\hline
\end{tabular}

The present motivation for this work is the numerical modeling of nonreflecting boundaries for the wave equation, discussed briefly here and in more detail in [16]. Maxwell's equations are also susceptible to similar treatment as outlined in [28]. The new representations enable the application of the exact nonreflecting boundary conditions, which are global in space and time, to be computationally effective.

\section{REFERENCES}

[1] D. Givoli. Non-reflecting boundary conditions. J. Comp. Phys., 94:1-29, 1991.

[2] B. Engquist and A. Majda. Absorbing boundary conditions for the numerical simulation of waves. Math. Comput., 31:629-651, 1977.

[3] A. Bayliss and E. Turkel. Radiation boundary conditions for wave-like equations. Comm. Pure Appl. Math., 23:707-725, 1980.

[4] M. Israeli and S. Orszag. Approximation of radiation boundary conditions.J. Comp. Phys., 41:115-135, 1981. 
[5] J.-P. Berenger. A perfectly matched layer for the absorption of electromagnetic waves. J. Comp. Phys., 114:185-200, 1994.

[6] I. L. Sofronov. Conditions for complete transparency on the sphere for the three-dimensional wave equation. Russian Acad. Sci. Dokl. Math., 46:397-401, 1993.

[7] M. J. Grote and J. B. Keller. Nonreflecting boundary conditions for time dependent scattering. J. Comp. Phys., 127:52, 1996.

[8] T. Hagstrom and S. I. Hariharan. A formulation of asymptotic and exact boundary conditions using local operators. Appl. Num. Math., to appear.

[9] T. Hagstrom, S. I. Hariharan, and R. C. MacCamy. On the accurate long-time solution of the wave equation in exterior domains: asymptotic expansions and corrected boundary conditions. Math. Comput., 63:507-539, 1994.

[10] T. Hagstrom. On high-order radiation boundary conditions. In B. Engquist and G. Kriegsmann, editors, IMA Volume on Computational Wave Propagation. Springer Verlag 1-22, 1996.

[11] J. R. Driscoll, D. M. Healy, and D. N. Rockmore. Fast discrete polynomial transforms with applications to data analysis for distance transitive graphs. SIAM J. Comput., 26:1066-1099, 1997.

[12] Martin J. Mohlenkamp. A fast transform for spherical harmonics, preprint, 1997.

[13] J. C. Nédélec. Quelques proprietes des dérivées logarithmiques des fonctions de hankel. Technical report, Ecole Polytechnique Centre od Mathematiques Appliquees, No. 259, 1992.

[14] A. Cruz and J. Sesma. Modulus and phase of the reduced logarithmic derivative of the hankel function. Math. Comput., 41:597-605, 1983.

[15] M. Abramowitz and I. A. Stegun, editors. Handbook of Mathematical Functions (10th printing). National Bureau of Standards (U.S.), Applied Mathematics Series 55, 1972.

[16] B. Alpert, L. Greengard, and T. Hagstrom. Nonreflecting boundary conditions for the time-dependent wave equation. To be submitted, J. Comp. Phys.

[17] F. W. J. Olver. Asymptotics and Special Functions. Academic Press, New York, 1974.

[18] F. W. J. Olver. The asymptotic expansion of bessel functions of large order. Philos. Trans. Roy. Soc. London, A247:328-368, 1954.

[19] F. W. J. Olver. The asymptotic solution of linear differential equations of the second order for large values of a parameter. Philos. Trans. Roy. Soc. London, A247:307-327, 1954.

[20] L. Greengard and V. Rokhlin. A fast algorithm for particle simulations. J. Comp. Phys., 73:325-348, 1987.

[21] C. R. Anderson. An implemenation of the fast multipole method without multipoles. SIAM J. Sci. Statist. Comput., 13:923-947, 1992.

[22] L. N. Trefethen. Rational Chebyshev approximation on the unit disk. Numer. Math., 37:297-320, 1981.

[23] M. H. Gutknecht and L. N. Trefethen. Real and complex Chebyshev approximation on the unit disk and interval. Bull. (New Ser.) Amer. Math. Soc., 8:455-458, 1983.

[24] M. H. Gutknecht. Rational Carathéodory-Fejér approximation on a disk, a circle, and an interval. $J$. Approx. Thy., 41:257-278, 1984.

[25] H. P. Starr. On the Numerical Solution of One-Dimensional Integral and Differential Equations. PhD thesis, Yale University, December, 1991.

[26] B. K. Alpert. Singularity-invariant Gauss-trapezoidal quadratures. To be submitted, SIAM J. Numer. Anal.

[27] J. Stoer and R. Bulirsch. Introduction to Numerical Analysis. Springer Verlag, 1980.

[28] T. Hagstrom, B. Alpert, L. Greengard, and S. I. Hariharan. Accurate boundary treatments for Maxwell's equations and their computational complexity. 14th Annual Review of Progress in Applied Computational Electromagnetics, 1998. 

